\title{
A Theory of Action for Classifying and Implementing Online Text- Based Curriculum Materials Using Natural Language Processing
}

\author{
Aaron M. Kessler, Anindya Roy, \& Daniel Seaton \\ Email: kesslera@mit.edu, anindyar@mit.edu, \& dseaton@mit.edu \\ Massachusetts Institute of Technology
}

\begin{abstract}
The purpose of this work is to propose a theory of action for how educators can use Natural Language Processing (NLP) as a way to explore, classify, and implement online text-based curricular materials. Grounded in previous research from k-12 mathematics and science education, the theory of action highlights the role of the educator in implementing curriculum materials. Within the context of open and free online text-based curricular materials, this work forwards and operationalizes the theory of action for using NLP in the context of higher education physics instruction. Results demonstrate the feasibility of such a model with implications for the importance of educators being "in the loop" instead of black boxing such processes.
\end{abstract}

\section{Significance of work}

Due to the complex nature and diversity of formal educational settings, research has repeatedly shown the impact of curricular materials on students' formal learning experiences depends on a number of factors associated with the educator (teacher, instructional designer, faculty member) and the materials themselves (Stein et al., 1996; Brown \& Edelson, 2003; Stigler \& Hiebert, 2004; Davis \& Krajcik, 2005; Forbes \& Davis, 2010). The increasing number of online curricular materials, especially open and free materials, presents a unique opportunity to think about and consider how educators organize and implement such distributed curriculum resources. Building on a number of frameworks from across mathematics and science education, we present a Theory of Action (TOA) that describes a mechanism by which educators can utilize trusted resources to train natural language processing (NLP) algorithms in order to better classify other sets of online resources that can be implemented by the educator. While we do not claim that this TOA is the only way such instructional work can be operationalized, we believe that by grounding our ideas in previous research we might leverage lessons learned and increase the speed and value in which the use of NLP and online materials can be more effectively implemented into the work of educators developing opportunities for students to learn.

\section{Theoretical background}

Traditionally, curriculum materials have come in the form of textbooks, workbooks, problem sets, lab manuals, and standards aligned assessments, often developed as products of publishing companies or resulting from projects with the explicit purpose of testing the impact of curricular materials or instructional interventions (Cervetti et al., 2015). Traditional curriculum materials were often designed with explicit learning goals tied to state or national standards and constructed to be used by an educator in purposeful sequences without the need for other curriculum resources. Further, these curricula "provide teachers with activities and tasks and then assume that they will simply deliver them to their students" (Beyer et al., 2009, pg.979).

One conception for organizing traditional curricular enactment in mathematics education, which has also been transformed into science education, is the Mathematical Task Framework (Stein et al., 2000; Cartier et al., 2013). Figure 1 shows a conceptualization of how curricular materials contain ideas as written that are set-up and organized by the educator, usually during the planning process, before being implemented in the classroom. 


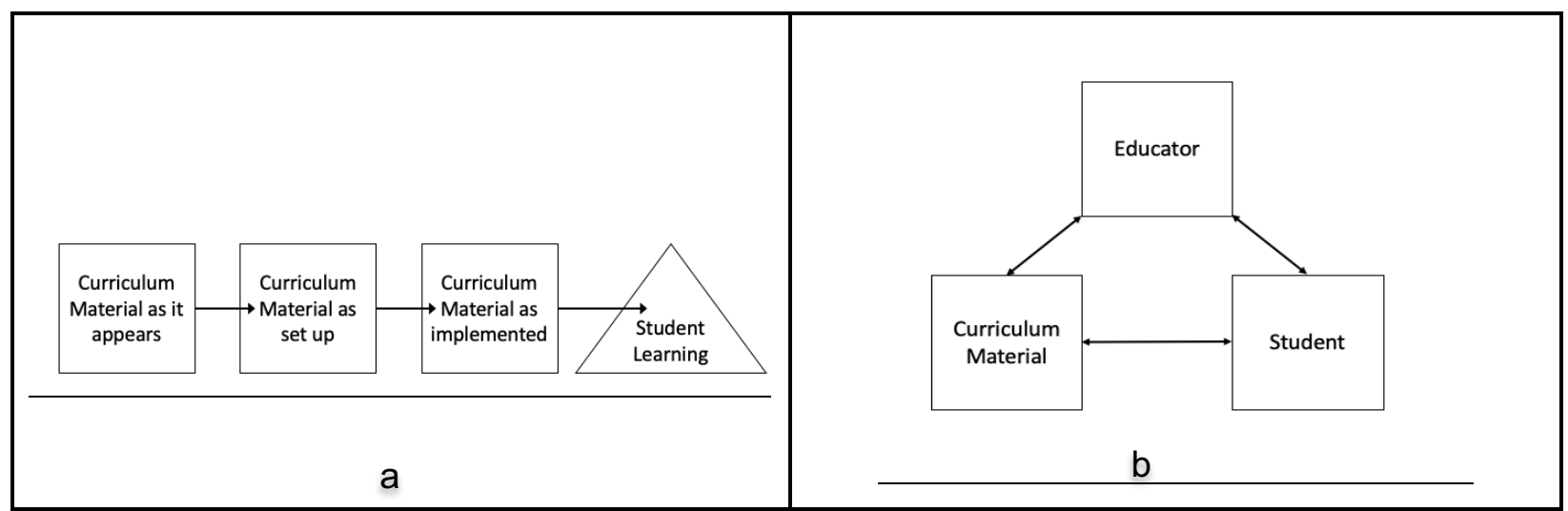

Figure 1. Mathematical Task Framework and Instructional Triangle

The first step in this process assumes that a high-quality set of curricular resources are in place. While vital for providing engaging and effective instruction, previous work shows that the implementation of those materials matters (Remillard, 2005; Lloyd \& Remillard, 2011; Davis et al., 2016). Across a number of educational settings traditional, reform-based, and technology mediated environments - the impact of educators' implementation of curricular materials on students' learning has been documented (Stein et al., 1996; Forbes \& Davis, 2010; Cai et al., 2013). Building on this work, the instructional triangle (Figure 2) makes clear that a student's opportunity to learn is actually related to the interaction between the curriculum materials, teacher, and student and not necessarily a linear output of the materials (Cohen, Raudenbush, \& Ball, 2003; Author 1 et al., 2014).

More recently, curriculum materials have begun to transform away from traditional forms and structures. Many materials have become digital, which has presented a number of opportunities and challenges. At the center of these digital materials are a new group of content creators who are leveraging the affordances of the internet and educational technologies to change how the educational community thinks about curriculum. This new generation includes the rise of a community focused on the production and release of open and free online resources for teachers and students, often created by those same teachers and students (Bliss \& Smith, 2017). This open educational resource (OER) community has also seen major contributions from parts of higher education including MIT's OpenCourseWare group and Rice University's OpenStax.

While the OER community produces more and more new content, the resources created by these groups are often far more distributed in nature as compared to more traditional curriculum. Unlike the traditional materials, OER curriculum materials have been less purposefully organized around learning goals and objectives or constructed to be implemented in specific sequences. In fact, it is likely that educators can no longer simply implement a single set of materials, instead needing to select and organize multiple sets of materials often situated across multiple platforms (YouTube, OCW, OpenStax). The particular challenge of scaling OER, and specifically text-based online resources, is the ability for educators to find resources that address the deeply contextualized instructional opportunities they are crafting for their students.

If educators are to take full advantage of the increasing number of text-based resources, and if the educational community at large is to understand how these scaled and widely distributed resources are utilized, the practitioner and research communities need ways to frame the expected actions educators take to find and implement such resources. In the sections that follow we suggest a potential theory of action that frames a mechanism for which educators can leverage natural language processing to address challenges associated with finding and classifying quality curricular resources and how, once classified, those materials can be taken up in similar ways as have been studied using traditional curriculum (Forbes and Davis, 2010; Brown, 2011).

\section{A theory of action for exploring curriculum using NLP}


The TOA presented in Figure 2 is predicated on a set of four assumptions. 1) The use of the term educators is intended to be very broad in the proposed TOA. Educators may be teachers, instructors, instructional designers, learning engineers, and others. Key to the proposed TOA is that multiple educators may play different parts in each of the proposed steps. It is also possible that a single educator will be responsible for the entire process. The role of the educator in the process will be completely dependent on the educational context in which the work is being conducted. 2) Educators know their context and the requirements needed of curricular materials beyond the one size fits all model of traditional curriculum. 3) Having sets of resources and processes with which educators can better design and develop learning opportunities for their students can produce better learning outcomes. 4) When processes associated with searching and categorizing curricular materials are black boxed the educators who use such resources risk being unable to interrogate their own work or how such processes might be improved. Based on these assumptions, Figure 2 presents a visual representation of the TOA for how educators can utilize NLP tools to train a model to classify online curricular resources in order to organize and implement such resources.

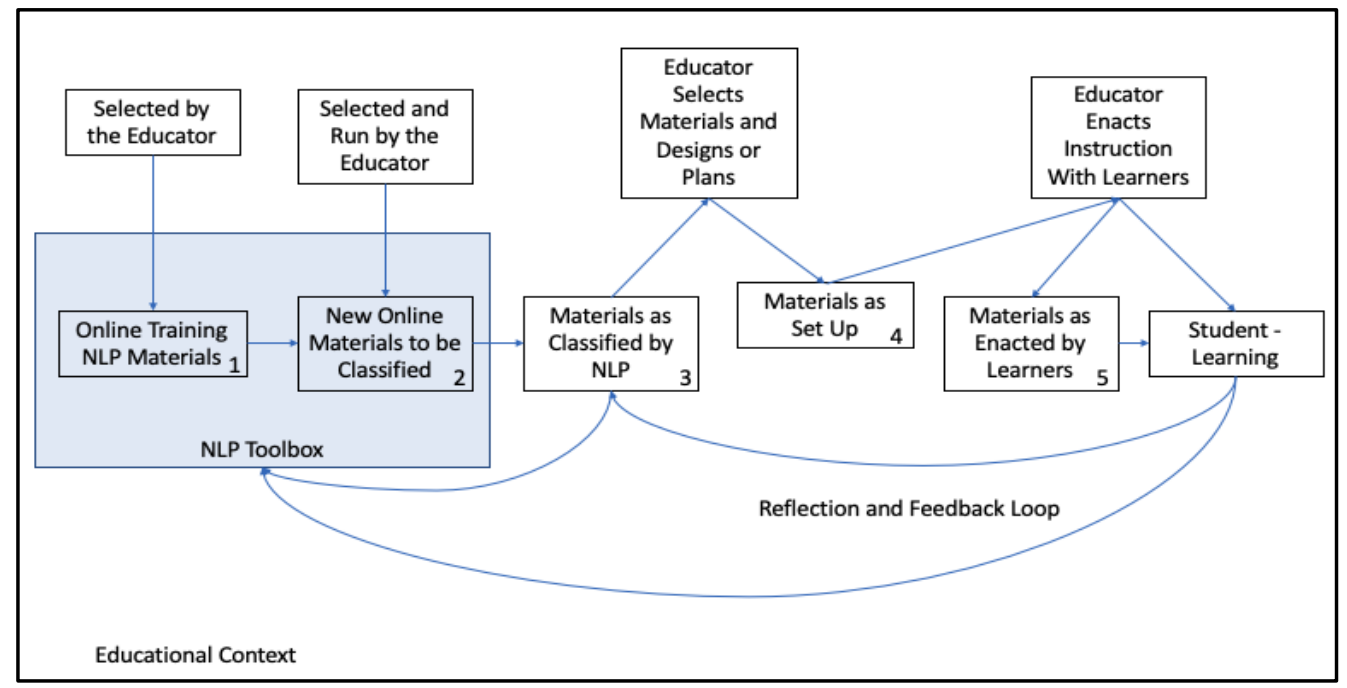

Figure 2. Theory of action for educators using NLP to classify and enact online-text based materials

The use of NLP for educational purposes is not particularly new and has the potential to provide solutions to the challenges faced by educators in finding and classifying resources they value (Kao \& Poteet, 2007). The two boxes $(1 \& 2)$ contained within the blue NLP toolkit box in Figure 2 are intended to represent how a set of NLP tools can be applied to online curriculum materials. The first step in the TOA involves the educator selecting a set of trusted or valued resources (Box 1) that can be used to train the NLP algorithm. The idea being that educators will already have some sets of resources or materials (online books, videos with transcripts, problem sets) that they value and would want to connect with other resources. As an example, if one is a high school chemistry teacher that uses an online textbook from a publisher that textbook can be used to initially train the NLP algorithms in a way that will allow other text-based materials to later be classified. Critical to this part of the TOA is that fact that the NLP tools are not searching out resources to classify. It is the educator who is responsible for feeding resources into the NLP tools. Having the educator know and understand the resources that are being used within the NLP tools is important for allowing them to engage in reflective practice on teaching and in improving NLP oversight (Baird et al., 1991).

Box 2 is meant to represent distributed online materials across a number of settings and contexts that need to be classified. Some of these materials have been developed by curriculum developers, some have been placed online with the express goal of sharing knowledge with the world (OCW), others have come to exist as parts of one-off projects with other goals (YouTube Videos), and some have been developed as content assessments (problem banks). No matter how they came to exist, these online materials can serve as important resources for educators attempting to find and implement curricular materials. However, they can only be used effectively if educators have the ability to classify and search these resources in purposeful ways, such as connecting them with resources that are already being 
used. As such, this part of the TOA represents the step in the process where educators apply the NLP trained classifying algorithm to the materials in order to produce a set of classified materials.

Box 3 represents the newly classified materials. Based on these classifications, the educator can now more easily find resources based on classifications that have a deeper meaning to them and their context. This, in theory, allows educators to streamline the process of finding resources that match the types of content and instruction they hope to design. It is the work of the educator to transition these materials from classified materials (Box 3 ) to a set of compiled resources that are purposefully organized by the educator for a specific instructional reason (Box 4). In this way, the "materials as classified by NLP" transition to "materials as set up" in similar ways as described in Figure 1a. When the materials move to Box 5 (Materials as Enacted by Learners) the enactment that produces potential learning mirrors student-educator-material interactions highlighted in Figure $1 \mathrm{~b}$.

In order to interrogate the beginning steps of this theory of action the work that follows is a demonstration of how the online material classification process of the TOA can be operationalized and the results of that work. Here we apply topic modeling and keyword extraction, both applications of NLP, as a means of exploring the first step of our TOA, the "NLP Toolbox".

\section{Methodological approach}

We grounded this exploration of applying NLP to resource classification in the content area of Physics, as it has a set of OER and two of the paper authors have content backgrounds in physics. We trained a topic model based on the OpenStax physics textbook "University Physics Vol. 1" (Moebs et al., 2016), to demonstrate how such a model could be used to identify topics from a set of OER with unknown topics. Then, as an example, we applied the OpenStax topic model to classify the following OER on circular motion:

- The Wikipedia article on circular motion (Wikipedia)

- A pdf document from an MIT Open Learning Library (MIT OLL) physics course (Mechanics)

- A transcript file of a 80-second video from the same course.

We use an implementation of Latent Dirichlet Allocation (LDA) (Blei et al., 2003) based on the popular NLP package gensim (Rehurek et al., 2010). LDA is a type of probabilistic modeling that infers hidden structure in a set of observable variables. In the case of a set of documents, the observed variables are words in documents and the topics are the hidden (latent) structure.

To train our model, we use the 106 individual sections from the OpenStax textbook as the documents. We included bigrams and trigrams (representing two- and three-word phrases), along with individual words from the documents processed through steps such as stopwords removal and lemmatization. We limited our N-grams to trigrams at most, as we found that higher $\mathrm{N}$-grams were seldom useful for capturing information from our corpus. To construct the bag of words for our model, we first constructed all possible bigrams and trigrams, and discarded those which occurred less than thrice in each document. Accepting bi-/trigrams which occurred at least thrice per document proved to be a reasonable compromise. We eliminated the bi-/trigrams with single occurrences since all bi-/trigrams would exist at least once in their source document. A large fraction of the bi-/trigrams which occurred exactly twice per document was found to be irrelevant for topic-extraction purposes, and hence we excluded them from consideration. To generate the bag of words, we iteratively collected all occurrences of a trigram or a bigram from a document in order of how frequently that Ngram appeared in the document. Once we accounted for all bi-/trigrams that occurred at least thrice in a document, we processed the rest of the document to extract the single words (unigrams) to include in the bag of words. On the second iteration of our LDA model, we consider only bi-/trigrams and exclude the unigrams.

As an unsupervised probabilistic model, the LDA-based algorithm takes the desired number of topics as a model input, and determines the topics as collections of words distributed over the documents. Any one document can have multiple topics, while a single topic could be present in multiple documents. Selecting a small number of topics results in leaving the corpus insufficiently resolved, with topics that are too broadly defined. Choosing a large number of topics, on the other hand, often leads to topics that are too similar and which contain overlapping terms. While there are mathematical measures such as coherence score to indicate how well a corpus is resolved into distinct topics 
(Chang et al., 2009; Mimno et al., 2011), the human interpretation of the set of topics may differ from what is mathematically optimal. In the Results section, we discuss how we use coherence score along with direct inspection of generated topics to arrive at reasonable model parameters, and apply that model on the unseen resources we wished to classify.

\section{Results}

The OpenStax University Physics Vol. 1 textbook has 106 sections, and we found the $c_{-} v$ coherence score (Syed et al., 2017) is maximized to about 0.48 with 21 topics. However, the topics in this set seemed overly broad. By increasing the number of topics to 50 , for example, we could achieve better separation among themes, although this resulted in lowering of the coherence score to 0.43 .

We compared this result with our second model where we excluded unigrams from the bag of words. The bi/trigram-based model has the highest coherence score of 0.52 for 94 topics. This is a relatively high number of topics compared to the previous model, and the associated coherence score is not much better. However, we found that the main improvement came from removing the unigrams: although we lost single-word identifiers related to the concepts (e.g., gravity), we managed to significantly cut down on words that are ubiquitous (e.g., car). In other words, we found that unigrams in our corpus have a low signal-to-noise ratio overall for the topic-modeling task. We stick to this bi/trigram-based model for the rest of the manuscript.

In Figure 3(a) we show the number of sections (documents) associated with each topic, and we see that most topics are uniquely tied to a single section or a couple of sections, indicating a relatively high section-level resolution of topical themes. The gaps corresponding to certain topic ids in Figure 3(a) indicate that those topics have less than 0.1 probability in the topic distribution of any section, and we excluded them from our plot for clarity. In Figure 3(b) we plotted the probability of a topic to appear in a section. We see that most sections are strongly connected with a small number of topics, as shown by dark red dots, which correspond to high topic probabilities in the topic distribution of a section. As in Figure 2(a), we excluded from this plot the topics which have topic-distribution probability less than 0.1 . These two figures together emphasize that each topic is related to a small number of sections and vice versa, with a few exceptions.
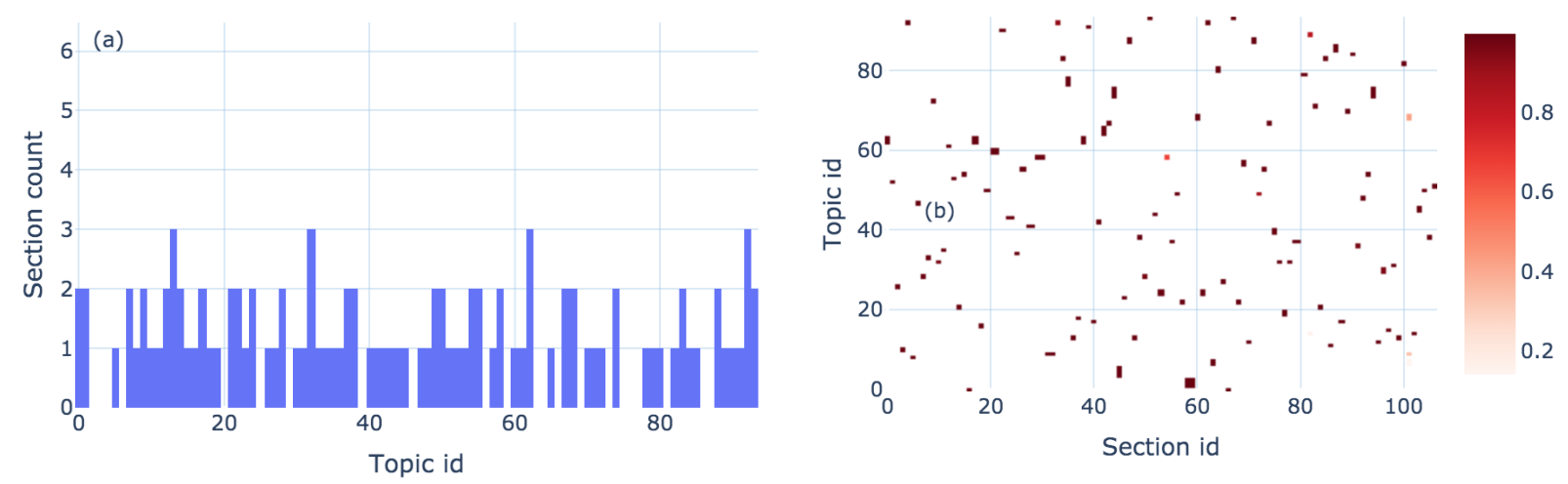

Figure 3. Topic modeling with LDA for the OpenStax Physics textbook. (a) Total number of sections for each topic, and (b) Distribution of 94 topics into 106 sections. The colorbar to the right corresponds to topic probability.

We found that 6 sections sections are examples of exceptions mentioned in the paragraph above, where the model failed to produce one-to-few associations between the topics and the sections. We looked at these sections individually and found that they are all from the appendix segment of the book, containing the periodic table of elements, astronomical data, fundamental physical constants, conversion factors, mathematical formulae, and the Greek alphabet. All these sections had little text left after the initial processing steps, and the model did not perform well in such a situation.

Once we trained our model with the OpenStax physics textbook, we applied this 94-topic model on the 3 unseen documents specified earlier. In Figure 4 we show the results schematically: the model associates the 
Wikipedia article titled "Circular Motion" with 2 topics, and the MIT OLL introductory text and the subtitle file on circular motion with one topic. The Wikipedia article is associated primarily with 2 topics, \#9 and \#88, with topicdistribution probabilities 0.54 and 0.25 respectively, while the text and the subtitle file from MIT OLL shows a very strong association with the topic $\# 9$ (a probability of 0.89 and 0.90 , respectively).

To put this into context, topic \#9 is associated with the sections "Uniform circular motion" and "Relating angular and translational quantities", and has the following bigrams and trigrams as its top-10 keywords: centripetal acceleration, tangential acceleration, circular motion, uniform circular motion, position vector, centripetal acceleration vector, total acceleration vector, velocity vector, toward center circle, nonuniform circular motion; Topic \#88 is strongly connected with the sections "Conditions for static equilibrium" and "Centripetal force", and the top-10 keywords for this topic are centripetal force, merry go round, inertial frame reference, frame reference, free body diagram, axis rotation, center gravity, equilibrium condition, banked curve, second equilibrium condition.

We included the short transcript file to test if the model assigns topics accurately, as short texts would contain few relevant bi-/trigrams. The model performed well on the transcript file (by identifying topic \#9 with a probability of 0.89 ), which led us to test it further on a very short problem text on circular motion from MIT OLL. Although this problem was on circular motion, the model failed to generate any strong association with any of the 94 topics. This exercise underscored the difficulty of inferring topics from short texts, as it depended precariously on generating enough keywords. This is especially true for our bi-/trigram-based model with a lower cutoff on keyword frequency, as the preprocessing of the short texts may eliminate the single occurrence of consequential phrases.

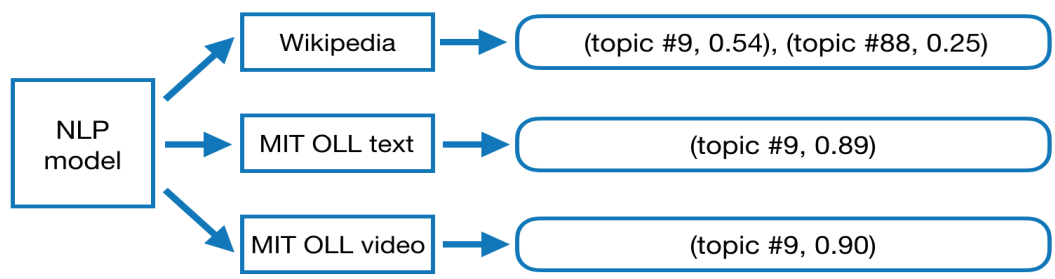

Figure4. Application of the NLP model on 3 specific resources and the inferred topics with associated topicdistribution probability

\section{Discussion}

This study indicates that even a simple model could perform reasonably well in classifying topics from unseen sources, if the text is not too short. The textbook that trained the model comes with associated features such as human-defined chapter names, keywords and learning objectives. Because of one-to-few connection between the sections and the topics (and vice versa), we can extend those attributes to unseen texts when we infer their topics using this model. In turn, such attributes allow us to tag the unseen documents with information beyond the topic keywords, and we can use them in knowledge-graph (Paulheim 2017) applications allowing resources to be organized in ways that likely have much more meaning to educators.

For college-level mechanics courses, it is common to see concepts of circular motion explained through examples of rides on a merry-go-round, or cars moving on a circular banked surface. Even if these contextual examples are not intrinsically tied to the concept of circular motion, topical themes emerged from our LDA-based models which relate conceptual terminologies (e.g., centripetal acceleration) with example terminologies (e.g., merry-go-round or banked curve). This poses a challenge while extracting topics from other short texts, where the dominant examplerelated terms are different from the examples used in the corpus, and the NLP model infers the topical themes based on the example terminology rather than the conceptual terminology. However, we expect such challenges to be overcome by extending the corpora to include different common examples.

We would like to emphasize that while topics can be extracted with reasonable fidelity, the open educational content from unverified sources still require expert supervision, and we draw attention to this aspect in Figure 2 with the arrow from "Materials as Classified by NLP" to "Educator Selects Materials and Designs or Plans". It is through this process that educators must leverage their pedagogical design capacity (Brown, 2011) to organize curriculum 
materials and plan instructional episodes that will afford students opportunities to actively engage with the content (Author, 2015).

\section{Conclusions and implications}

By making the TOA explicit this work hopes to provide the NLP and Learning Sciences communities with a frame for thinking about the next steps in exploring, understanding, and testing how educators can and should engage with NLP as a way to find and classify online text-based curricular resources. In grounding this work in research related to curriculum material implementation, we hope to point researchers and educators to critical portions of the TOA that demand attention in support of learners and learning outcomes. Most importantly, the education community must move beyond the black box approach to curriculum in order to make explicit how we conduct the work of finding, organizing, and enacting distributed curricular materials. More research is needed in order to build effective NLP toolkits for educators and to understand how educators implement such tools.

While this work provides one specific case of how tertiary physics educators might take up the NLP classification step of the theory of action, many different groups of educators that come from a number of content areas and grade levels are likely interested in such work. Some of these groups include higher education instructors, secondary content specific teachers, general k-12 teachers, instructional designers, library service groups, and other emergent subject areas (e.g., Materials Science). Understanding the range of educators will be critical as researchers and developers decide on how to design the NLP toolbox as well as structuring research opportunities to understand similarities and differences in how educators will value NLP classifications and enact such materials.

Lastly, while considering the individual parts of the TOA for research purposes can be a valuable first step, we must interrogate the TOA model holistically to fully understand how the interactions across all parts, situated in complex settings, impact students opportunities to learn. The next step in our work will focus on studying how a group of higher education course developers leverage these NLP based process with the goal of providing important insights into next steps for refining and understanding the proposed theory of action.

\section{References}

Baird, J. R., Fensham, P. J., Gunstone, R. F., \& White, R. T. (1991). The importance of reflection in improving science teaching and learning. Journal of research in Science Teaching, 28(2), 163-182.

Beyer, C. J., Delgado, C., Davis, E. A., \& Krajcik, J. (2009). Investigating teacher learning supports in high school biology curricular programs to inform the design of educative curriculum materials. Journal of Research in Science Teaching: The Official Journal of the National Association for Research in Science Teaching, 46(9), 977-998.

Blei, D. (2017). Scalable Topic Modeling: Online Learning, Diagnostics, and Recommendation. COLUMBIA UNIV NEW YORK NEW YORK United States. [web]

Blei, D. M., Ng, A. Y., \& Jordan, M. I. (2003). Latent dirichlet allocation. Journal of machine Learning research, 3(Jan), 993-1022.

Bliss, T. J., \& Smith, M. (2017). A brief history of open educational resources. Open: The philosophy and practices that are revolutionizing education and science, 9-27.

Brown, M. W. (2011). The teacher-tool relationship: Theorizing the design and use of curriculum materials. In Mathematics teachers at work (pp. 37-56). Routledge.

Brown, M. \& Edelson, D. (2003). Teaching as design: Can we better understand the ways in which teachers use materials so we can better design materials to support their changes in practice? (RS-03). Evanston, IL: The Center for Learning Technologies in Urban Schools.

Cai, J., Moyer, J. C., Wang, N., Hwang, S., Nie, B., \& Garber, T. (2013). Mathematical problem posing as a measure of curricular effect on students' learning. Educational Studies in Mathematics, 83(1), 57-69.

Cartier, J. L., Smith, M. S., Stein, M. K., \& Ross, D. K. (2013). 5 Practices for Orchestrating Productive Science Discussions. Reston, VA: National Council of Teachers of Mathematics and Corwin Press. 
Cervetti, G. N., Kulikowich, J. M., \& Bravo, M. A. (2015). The effects of educative curriculum materials on teachers' use of instructional strategies for English language learners in science and on student learning. Contemporary Educational Psychology, 40, 86-98.

Chang, J., Gerrish, S., Wang, C., Boyd-Graber, J. L., \& Blei, D. M. (2009). Reading tea leaves: How humans interpret topic models. In Advances in neural information processing systems (pp. 288-296).

Cohen, D. K., Raudenbush, S. W., \& Ball, D. L. (2003). Resources, instruction, and research. Educational evaluation and policy analysis, 25(2), 119-142.

Davis, E. A., Janssen, F. J., \& Van Driel, J. H. (2016). Teachers and science curriculum materials: Where we are and where we need to go. Studies in Science Education, 52(2), 127-160.

Davis, E.A. \& Krajcik, J. (2005). Designing educative curriculum materials to promote teacher learning. Educational Researcher, 34(3), 3-14.

Forbes, C. T., \& Davis, E. A. (2010). Curriculum design for inquiry: Preservice elementary teachers' mobilization and adaptation of science curriculum materials. Journal of Research in Science Teaching, 47(7), 820-839.

Kao, A., \& Poteet, S. R. (Eds.). (2007). Natural language processing and text mining. Springer Science \& Business Media.

Litman, D. (2016, March). Natural language processing for enhancing teaching and learning. In Thirtieth AAAI Conference on Artificial Intelligence.

Lloyd, G. M. \& Remillard, J.T. (2011). Teachers' use of curriculum materials: An emerging field. In Mathematics teachers at work (pp. 23-34). Routledge.

Mechanics: Kinematics and Dynamics (2019). Retrieved from https://openlearninglibrary.mit.edu/

Mimno, D., Wallach, H. M., Talley, E., Leenders, M., \& McCallum, A. (2011, July). Optimizing semantic coherence in topic models. In Proceedings of the conference on empirical methods in natural language processing (pp. 262-272). Association for Computational Linguistics.

Moebs, W., Ling, S. J., \& Sanny, J. (2016). University Physics Volume 1. Available for free at https://openstax.org/books/university-physics-volume-1/pages/1-introduction

Newman, D., Lau, J. H., Grieser, K., \& Baldwin, T. (2010, June). Automatic evaluation of topic coherence. In Human Language Technologies: The 2010 Annual Conference of the North American Chapter of the Association for Computational Linguistics (pp. 100-108). Association for Computational Linguistics.

Paulheim, H. (2017). Knowledge graph refinement: A survey of approaches and evaluation methods. Semantic web, $8(3), 489-508$.

Rehurek, R., \& Sojka, P. (2010). Software framework for topic modelling with large corpora. In In Proceedings of the LREC 2010 Workshop on New Challenges for NLP Frameworks.

Remillard, J. T. (2005). Examining Key Concepts in Research on Teachers' Use of Mathematics Curricula. Review of Educational Research, 75(2), 211-246.

Smith, M. S., \& Stein, M. K. (2011). 5 Practices for Orchestrating Productive Mathematics Discussions. Reston, VA: National Council of Teachers of Mathematics and Corwin Press.

Stein, M. K., Engle, R. A., Smith, M. S., \& Hughes, E. K. (2008). Orchestrating productive mathematical discussions: Five practices for helping teachers move beyond show and tell. Mathematical Thinking and Learning, 10, 313-340.

Stein, M. K., Grover, B. W., and Henningsen, M. (1996). Building student capacity for mathematical thinking and reasoning: An analysis of mathematical tasks used in reform classrooms. American Educational Research Journal, 33, 455-488.

Stein, M. K., Smith, M.S., Henningsen, M., \& Silver, E.A. (2000). Implementing standards-based mathematics instruction: A casebook for professional development. New York: Teachers College Press.

Stigler, J. W., \& Hiebert, J. (2004). Improving mathematics teaching. Educational leadership, 61(5), 12-17.

Syed, S., \& Spruit, M. (2017, October). Full-text or abstract? Examining topic coherence scores using latent dirichlet allocation. In 2017 IEEE International conference on data science and advanced analytics (DSAA) (pp. 165174). IEEE.

Wikipedia: Circular Motion (n.d.). Retrieved from https://en.wikipedia.org/wiki/Circular_motion 